\title{
Expanding Quark-Gluon Plasmas: Transverse Flow, Chemical Equilibration and Electromagnetic Radiation
}

\author{
Dinesh Kumar Srivastava, Munshi Golam Mustafa \\ Variable Energy Cyclotron center, 1/AF Bidhan Nagar, Calcutta 700064 \\ Berndt Müller \\ Department of Physics, Duke University, Durham, North Carolina 27708-0305
}

(November 11, 2018)

\begin{abstract}
We investigate the chemical equilibration of the parton distributions in collisions of two heavy nuclei. We use initial conditions obtained from a self-screened parton cascade calculation and, for comparison, from the HIJING model. We consider a one-dimensional, as well as a three-dimensional expansion of the parton plasma and find that the onset of the transverse expansion impedes the chemical equilibration. At energies of $100 \mathrm{GeV} /$ nucleon, the results for one-dimensional and three-dimensional expansion are quite similar except at large values of the transverse radius. At energies of several $\mathrm{TeV} /$ nucleon, the plasma initially approaches chemical equilibrium, but then is driven away from it, when the transverse velocity gradients develop. We find that the total parton multiplicity density remains essentially unaffected by the flow, but the individual concentrations of quarks, antiquarks, and gluons are sensitive to the transverse flow. The consequences of the flow are also discernible in the transverse momenta of the partons and in the lepton pair spectra, where the flow causes a violation of the so-called $M_{T}$ scaling.
\end{abstract}

\section{INTRODUCTION}

Relativistic heavy ion collisions are being studied with the intention of investigating the properties of ultradense, strongly interacting matter. A central theme of this area of research is the search for a quark-gluon plasma (QGP) [1]. Extensive theoretical studies done over the last several years for nuclear reactions at collider energies have helped develop a picture of the colliding nuclei as two clouds of valence and sea partons which interact passing through each other [2]. Partonic final-state interactions are then thought to produce a dense plasma of quarks and gluons. This plasma expands and becomes cooler and more dilute. If quantum chromodynamics admits a first-order deconfinement or chiral phase transition, this plasma will pass through a mixed phase of quarks, gluons, and hadrons, before the hadrons lose thermal contact and stream freely towards the detectors.

What is the structure of the matter formed in these nuclear collisions? Does the initial partonic system attain kinetic equilibrium? Probably yes, as the initial parton density is large, forcing the partons to suffer many collisions in a very short time [3]. Does it attain chemical equilibration? This will depend on the time available [4] to the partonic system before it converts into a mixed phase, or before perturbative QCD is no longer applicable. The time available for equilibration is perhaps too short $(3-5 \mathrm{fm} / \mathrm{c})$ at the energies $(\sqrt{s} \leq 100$ $\mathrm{GeV} /$ nucleon) accessible to the Relativistic Heavy Ion Collider (RHIC). At the energies $(\sqrt{s} \leq 3 \mathrm{TeV} /$ nucleon $)$ that will be achieved at the CERN Large Hadron Collider (LHC) this time could be large (more than $10 \mathrm{fm} / c$ ). If one considered only a longitudinal expansion of the system, the QGP formed at LHC energies could approach chemical equilibrium very closely, due to the higher initial temperature predicted to be attained there. However, then the life-time of the plasma would also be large enough to allow a rarefaction wave from the surface of the plasma to propagate to the center. As we shall discuss below, the large transverse velocity gradients developing in this process at LHC energies may drive the system away from the chemical equilibrium [9].

It is evident that the evolution of the partonic system will depend on the initial conditions and the total time available to the system where the partonic picture still holds. In order to study this aspect, we use initial conditions from two models: the Self-Screened Parton Cascade (SSPC) model 10 and the HIJING model [11.

By now, a considerable progress has been achieved in our understanding of the parton cascades which develop in the wake of the collisions. Early calculations [2] were done by assuming fixed $p_{T^{-}}$and virtuality cut-offs for the partonic interactions to ensure the applicability of the perturbative expansion for QCD scattering processes. In the recently formulated self-screened parton cascade model early hard scatterings produce a medium which screens the longer ranged color fields associated with softer interactions. When two heavy nuclei collide at sufficiently high energy, the screening occurs on a length scale where perturbative QCD still applies. This approach yields predictions for the initial conditions of the forming QGP without the need for any ad-hoc momentum and virtuality cut-off parameters. These calculations also show that the QGP likely to be formed in such collisions could be very hot and initially far from chemical equilibrium.

It is still useful to explore the dependence of the course of evolution of the plasma on the initial conditions. For this purpose we use the results of the HIJING Monte Carlo model which combines multiple hard or semihard parton scatterings with initial- and final-state 
radiation with Lund string phenomenology for the accompanying soft nonperturbative interactions to describe nuclear interactions. The uncertainties of the model are parametrized by allowing for variations of the initial fugacities. This approach has, for example, been used recently [12] in connection with the suppression of $\mathrm{J} / \psi$ in an equilibrating partonic plasma.

In the next section we briefly describe the hydrodynamic and chemical evolution of the plasma in a $(1+1)$ dimensional longitudinal expansion and a $(3+1)$ dimensional transverse expansion. The thermal photon and thermal lepton pair production is discussed in Section III, followed by a summary in Section IV.

\section{HYDRODYNAMIC EXPANSION AND CHEMICAL EQUILIBRATION}

\section{A. Basic Equations}

We start with the assumption that the system achieves kinetic equilibrium by the time $\tau_{i}$. We define the beginning of this epoch as the instant beyond which the momenta of the partons become locally isotropic. Local isotropy here is defined as the coincidence of the exponential slopes of the longitudinal and transverse momentum distributions, when they are integrated over a comoving volume with linear dimensions of one partonic mean free path $\lambda_{f}$. This occurs after a proper time of about $0.7 \lambda_{f}$ [3], corresponding to $\tau_{i} \approx 0.2-0.3 \mathrm{fm} / c$ under the conditions expected at collider energies [4]. Beyond this point, further expansion can be described by hydrodynamic equations. The approach to the chemical equilibration is then governed by a set of master equations which are driven by the two-body reactions $(g g \leftrightarrow q \bar{q})$ and gluon multiplication and its inverse process, gluon fusion $(g g \leftrightarrow g g g)$. The hot matter continues to expand and cools due to expansion and chemical equilibration. Once the energy density reaches a critical value (here taken as $\left.\epsilon_{f}=1.45 \mathrm{GeV} / \mathrm{fm}^{3}[13]\right)$ we terminate the evolution before entering into the hadronization phase.

Thus the expansion of the system is described by the equation for conservation of energy and momentum of an ideal fluid:

$$
\partial_{\mu} T^{\mu \nu}=0, \quad T^{\mu \nu}=(\epsilon+P) u^{\mu} u^{\nu}+P g^{\mu \nu},
$$

where $\epsilon$ is the energy density and $P$ is the pressure measured in the frame comoving with the fluid. The fourvelocity vector $u^{\mu}$ of the fluid satisfies the constraint $u^{2}=-1$. For a partially equilibrated plasma of massless particles, the equation of state can be written as [4]

$$
\epsilon=3 P=\left[a_{2} \lambda_{g}+b_{2}\left(\lambda_{q}+\lambda_{\bar{q}}\right)\right] T^{4},
$$

where $a_{2}=8 \pi^{2} / 15, b_{2}=7 \pi^{2} N_{f} / 40, N_{f} \approx 2.5$ is the number of dynamical quark flavors, and $\lambda_{k}$ is the fugacity for the parton species $k$. Here we have defined the fugacities through the relations,

$$
n_{g}=\lambda_{g} \tilde{n}_{g}, \quad n_{q}=\lambda_{q} \tilde{n}_{q}
$$

where $\tilde{n}_{k}$ is the equilibrium density for the parton species $k$ :

$$
\begin{gathered}
\tilde{n}_{g}=\frac{16}{\pi^{2}} \zeta(3) T^{3}=a_{1} T^{3}, \\
\tilde{n}_{q}=\frac{9}{2 \pi^{2}} \zeta(3) N_{f} T^{3}=b_{1} T^{3} .
\end{gathered}
$$

We further assume that $\lambda_{q}=\lambda_{\bar{q}}$. The equation of state (2) implies the speed of sound $c_{s}=1 / \sqrt{3}$.

We solve the hydrodynamic equations (1) with the assumption that the system undergoes a boost invariant longitudinal expansion along the $z$-axis and a cylindrically symmetric transverse expansion 14. It is then sufficient to solve the problem for $z=0$, because of the assumption of boost invariance.

The master equations [ 4 ] for the dominant chemical reactions $g g \leftrightarrow g g g$ and $g g \leftrightarrow q \bar{q}$ are

$$
\begin{aligned}
& \partial_{\mu}\left(n_{g} u^{\mu}\right)=n_{g}\left(R_{2 \rightarrow 3}-R_{3 \rightarrow 2}\right)-\left(n_{g} R_{g \rightarrow q}-n_{q} R_{q \rightarrow g}\right), \\
& \partial_{\mu}\left(n_{q} u^{\mu}\right)=\partial_{\mu}\left(n_{\bar{q}} u^{\mu}\right)=n_{g} R_{g \rightarrow q}-n_{q} R_{q \rightarrow g},
\end{aligned}
$$

in an obvious notation.

If we assume the system to undergo a purely longitudinal boost invariant expansion, (11) reduces to the well known relation 15

$$
\frac{d \epsilon}{d \tau}+\frac{\epsilon+P}{\tau}=0,
$$

where $\tau$ is the proper time. This equation implies

$$
\epsilon \tau^{4 / 3}=\text { const. }
$$

and the chemical master equations reduce to 4

$$
\begin{aligned}
& \frac{1}{\lambda_{g}} \frac{d \lambda_{g}}{d \tau}+\frac{3}{T} \frac{d T}{d \tau}+\frac{1}{\tau}=R_{3}\left(1-\lambda_{g}\right)-2 R_{2}\left(1-\frac{\lambda_{q} \lambda_{\bar{q}}}{\lambda_{g}^{2}}\right) \\
& \frac{1}{\lambda_{q}} \frac{d \lambda_{q}}{d \tau}+\frac{3}{T} \frac{d T}{d \tau}+\frac{1}{\tau}=R_{2} \frac{a_{1}}{b_{1}}\left(\frac{\lambda_{g}}{\lambda_{q}}-\frac{\lambda_{\bar{q}}}{\lambda_{g}}\right)
\end{aligned}
$$

which are then solved numerically for the fugacities. The rate constants $R_{2}$ and $R_{3}$ are related to the rates appearing in (6) and are given by [4]

$$
\begin{aligned}
& R_{2} \approx 0.24 N_{f} \alpha_{s}^{2} \lambda_{g} T \ln \left(1.65 / \alpha_{s} \lambda_{g}\right), \\
& R_{3}=1.2 \alpha_{s}^{2} T\left(2 \lambda_{g}-\lambda_{g}^{2}\right)^{1 / 2},
\end{aligned}
$$

where the color Debye screening and the LandauPomeranchuk-Migdal effect suppressing the induced gluon radiation have been taken into account, explicitly.

In case of transverse expansion, the master equations can be shown [9] to lead to partial differential equations: 


$$
\begin{aligned}
\frac{\gamma}{\lambda_{g}} \partial_{t} \lambda_{g} & +\frac{\gamma v_{r}}{\lambda_{g}} \partial_{r} \lambda_{g}+\frac{1}{T^{3}} \partial_{t}\left(\gamma T^{3}\right)+\frac{v_{r}}{T^{3}} \partial_{r}\left(\gamma T^{3}\right) \\
& +\gamma \partial_{r} v_{r}+\gamma\left(\frac{v_{r}}{r}+\frac{1}{t}\right) \\
& =R_{3}\left(1-\lambda_{g}\right)-2 R_{2}\left(1-\frac{\lambda_{q} \lambda_{\bar{q}}}{\lambda_{g}^{2}}\right), \\
\frac{\gamma}{\lambda_{q}} \partial_{t} \lambda_{q} & +\frac{\gamma v_{r}}{\lambda_{q}} \partial_{r} \lambda_{q}+\frac{1}{T^{3}} \partial_{t}\left(\gamma T^{3}\right)+\frac{v_{r}}{T^{3}} \partial_{r}\left(\gamma T^{3}\right) \\
& +\gamma \partial_{r} v_{r}+\gamma\left(\frac{v_{r}}{r}+\frac{1}{t}\right) \\
& =R_{2} \frac{a_{1}}{b_{1}}\left(\frac{\lambda_{g}}{\lambda_{q}}-\frac{\lambda_{\bar{q}}}{\lambda_{g}}\right)
\end{aligned}
$$

where $v_{r}$ is the transverse velocity and $\gamma=1 / \sqrt{1-v_{r}^{2}}$. It is easy to verify that the equations (11) reduce to (9) in the absence of transverse expansion.

The hydrodynamic equations (11) are solved numerically to get $\epsilon(r, t)$ and $v_{r}(r, t)$, which serve as input into the equations (11) for the fugacities. In all our solutions we have assumed that the initial transverse velocities are zero. We have verified that our results near $r=0$ closely follow the results for a purely longitudinal expansion, till the time when the fluid is disturbed by the rarefaction wave traveling from the surface to the center.

\section{B. Results for RHIC Energies}

In the following, we shall discuss the results obtained for the initial conditions listed in Table I, which are predicted for a central collision of gold nuclei. We shall discuss the results for the initial conditions from SSPC calculations in some detail, and then present the final results for the initial conditions from the HIJING model.

Let us first look at conditions likely to prevail at RHIC energies. For the case of a boost-invariant and a purely longitudinal expansion, the results are discussed in the literature 16. Here we concentrate on the question, how the transverse expansion of the plasma affects the chemical equilibration, and how the consequences of the transverse flow can best be identified.

Recall that for a boost-invariant longitudinal expansion, Eq.(11) provides that $\epsilon \tau^{4 / 3}$ is a constant (8). Let us look at the constant energy density contours,

$$
\epsilon(r, t)=\epsilon_{i} / N^{4 / 3}
$$

for $N=1,2,3, \ldots, 16$ (Figure 1a). The choice of hypersurfaces of equal comoving energy density is natural, because it interpolates smoothly between the initial condition and the final hadronization hypersurface, where the quark-gluon plasma freezes out into hadrons. If there were no transverse expansion, all these contours would be parallel to the line for $N=1$ and given by

$$
\tau=N \tau_{i} ; \quad \text { (longitudinal flow only), }
$$

extending up to $r=R_{T}$.

For the initial energy density assumed at RHIC, $N=16$ corresponds to an energy density of about 1.5 $\mathrm{GeV} / \mathrm{fm}^{3}$, which coincides with our choice of the density $\epsilon_{f}$ where the plasma finally hadronizes. We have also shown a line $r=R_{T}-c_{s} t$ which indicates the radial size of the region not yet affected by the rarefaction wave at any given time $t$. We see that, as expected, the fluid beyond $r=4 \mathrm{fm}$ is likely to be affected by the flow, by the time the system has cooled to the edge of the QGP phase.

In Fig. 1b-d we have given the gluon fugacity, the quark fugacity, and the transverse velocity along these contours. We see that deviations from the expectations for a longitudinal expansion [16], which we closely reproduce for small $r$, have a clear origin in the growth of the transverse velocity.
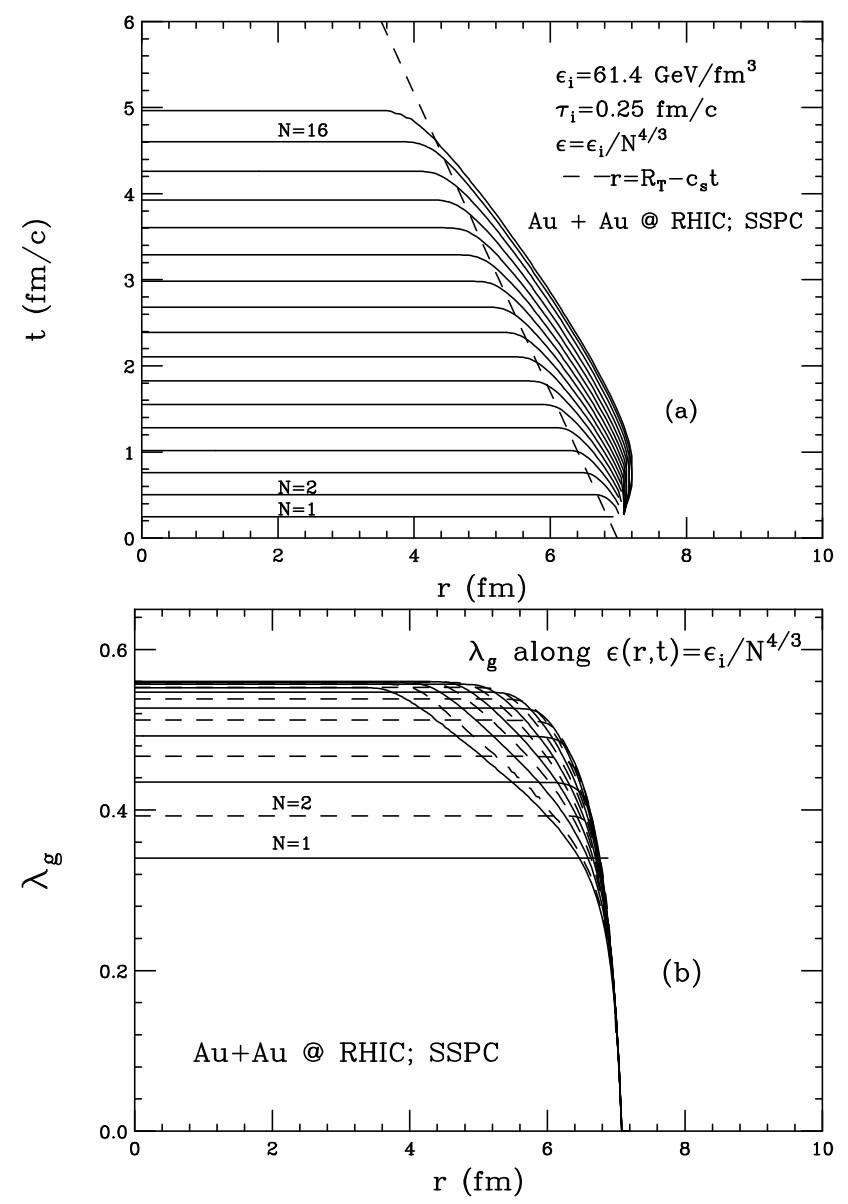

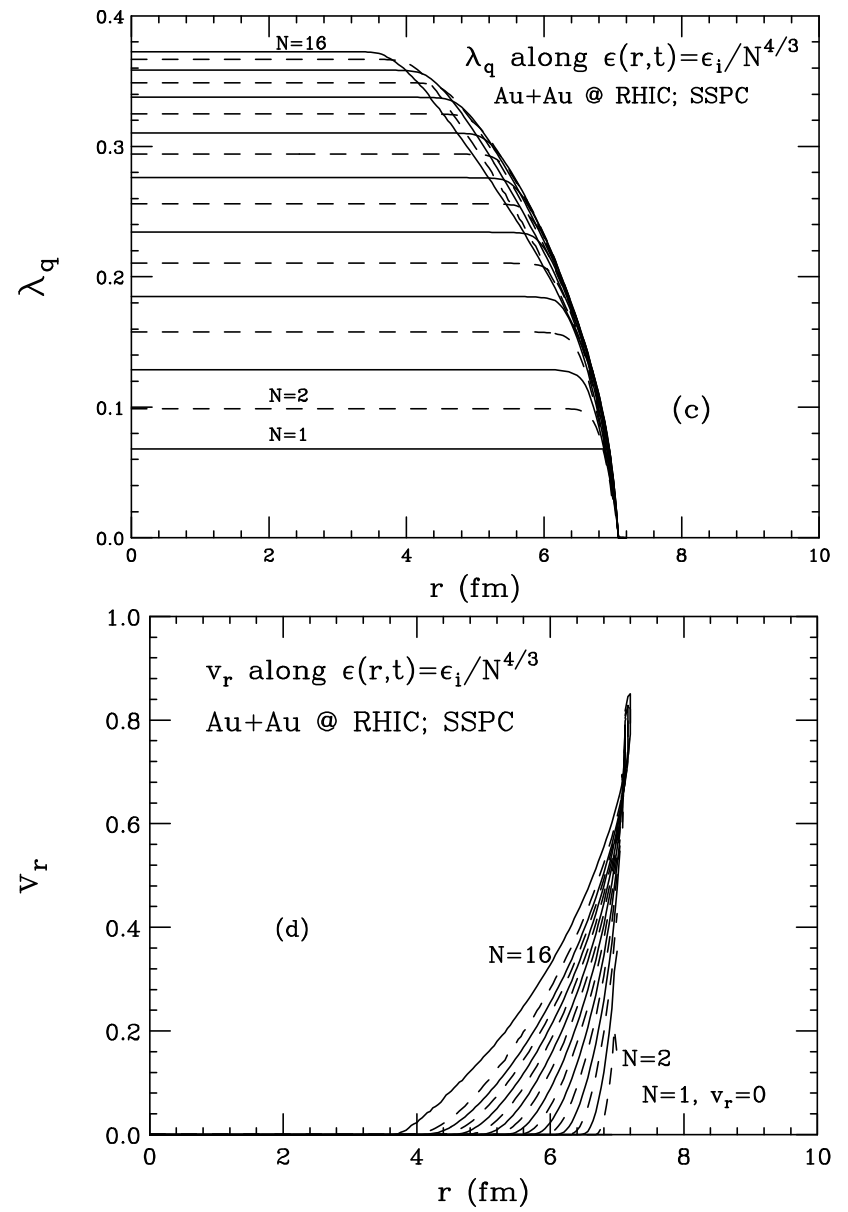

FIG. 1. (a) Constant energy density contours for a transversely expanding quark-gluon plasma likely to be created in $\mathrm{Au}+\mathrm{Au}$ collisions at RHIC. For a boost invariant longitudinal expansion these contours would be equidistant lines parallel to $N=1$. The dashed line gives the distance from the axis where the rarefaction wave has arrived at time $t$, and beyond which the fluid is strongly affected by the flow. (b) Gluon fugacity along the constant energy density contours. (c) Quark fugacities along the constant energy density contours. (d) Transverse velocities along the constant energy density contours.

For small values of $N$, we see that the plasma at the center has more time at its disposal to develop while at large values of $r$ the time available to the plasma is shorter, leading to a decrease in the fugacities (at constant $\epsilon$ ) as $r$ increases. However, this is not the most interesting observation. We see a unique feature, which is quite evident at large $r$ but is beginning to emerge at smaller $r$ as well, especially for the gluon fugacity: The fugacities initially increase with $N$, but then start decreasing. Thus the region where transverse velocities are large, the plasma ends its journey further away from chemical equilibrium, as compared to the region where the transverse velocity is still small. We shall see later that this has its origin in the velocity gradients which develop in the fluid.

This has an interesting consequence, namely, the plasma nearer to the surface will be hotter than in the interior, for a given energy density. If we make the reasonable assumption that the transition point between the quark-gluon plasma and the hadron phase is determined by a certain critical value of the energy density, then our result implies that the outer regions of the cylindrical reaction zone hadronize at a higher temperature than the inner region. In other words, it is quite likely that the hadronic matter may be formed at different temperatures in different regions. This enhances the complexity of the final state produced in the hydrodynamic model compared to what was expected earlier.

How does the flow affect the momentum distribution of the partons? We can evaluate the evolution of the parton distributions with time for the longitudinal expansion, and compare it with the corresponding results for the transverse expansion, using the Cooper-Frye formula 17,18];

$$
\frac{d N}{d^{3} \vec{p} / E}=\frac{d N}{d^{2} p_{T} d y}=\frac{g}{(2 \pi)^{3}} \int_{\sigma} f(x, p) p^{\mu} d \sigma_{\mu},
$$

where $f(x, p)$ is the phase-space distribution of the parton, $g$ is the color, spin, and flavor degeneracy, and $\sigma$ is the surface described by the contours given by (12) or (13). For massless particles and in the absence of transverse flow simplifications arise, which we shall not discuss here [18].

The resulting transverse momentum spectra for gluons and quarks is shown in Figs. 2a-b. We see that the parton distributions at large $p_{T}$ are affected strongly as the flow develops. It is known that if the plasma were to undergo a first order phase transition, then the nonvanishing transverse velocity at the beginning of the mixed phase will reduce its life-time. We shall see, however, that due to several other competing factors, like lower temperature at later times, the flow only marginally affects the production of thermal photons and lepton pairs from the quark-gluon phase at RHIC energies. The presence of significant collective transverse flow is clearly visible in the positive curvature of the $p_{T}$-spectra at late times $(N=12,16)$. The slope of these spectra at large $p_{T}$, resulting from a superposition of thermal and collective motion, agrees remarkably well with the homogeneous slope of the initial spectrum $(N=1)$ and thus serves as an indicator of the very high apparent temperature achieved at the moment of thermalization. 

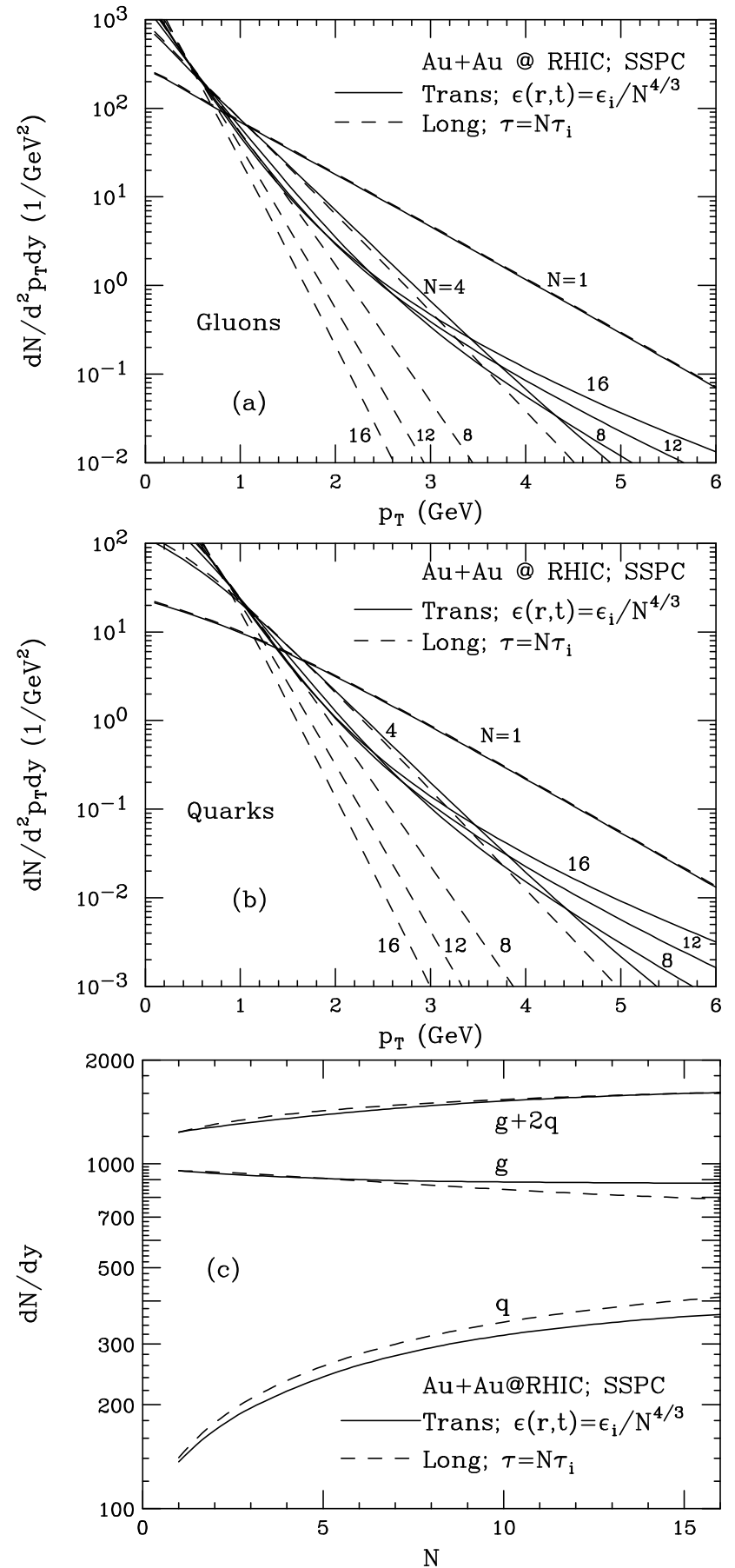

FIG. 2. Evolution of the momentum distributions of (a) gluons and (b) quarks, and (c) of the multiplicity of partons with (solid curves) and without (dashed curves) transverse flow at RHIC.

We can also evaluate the multiplicity density of the partons as a function of $N$ to see the increase in entropy, as the chemical equilibration proceeds, and investigate its dependence on the flow. We see from Fig. 2c that the total entropy at the end of the QGP phase is essentially identical for the scenarios with and without transverse flow. We also see that as the chemical equilibration is accompanied by a steady increase in the net number of quarks (and antiquarks) whereas the number of gluons decreases.

However, the detailed composition of the partonic matter at the end of the QGP phase is different for the case with flow. We have approximately $12 \%$ less quarks and antiquarks but about 10\% more gluons compared to our result without flow. It is difficult to predict how these rather subtle differences in the composition of the plasma will affect the final particle yields. We may speculate that an increased gluon abundance could result in the enhanced production of hadrons with a significant "valence-glue" component, such as $\eta$ - and $\eta^{\prime}$-mesons or even glueballs.

\section{Results for LHC Energies}

Let us next turn to the results for $\mathrm{Au}+\mathrm{Au}$ collisions at LHC energies, some of which have been reported earlier [9]. In Figs. 3a-d, we present the constant energy density contours, the fugacities for gluons and quarks, and the transverse velocities. The representation is the same as the one for the RHIC-related figures, except that we have plotted only a reduced number of equal-energy contours. Due to the very large number of contours between the initial energy density and the hadronization density $\epsilon_{f}=1.45 \mathrm{GeV} / \mathrm{fm}^{3}$ we have plotted only the contours $N=1,6,11, \ldots, 71$ in the energy density plot and $N=1,11,21, \ldots, 71$ in the plots for the other three quantities.

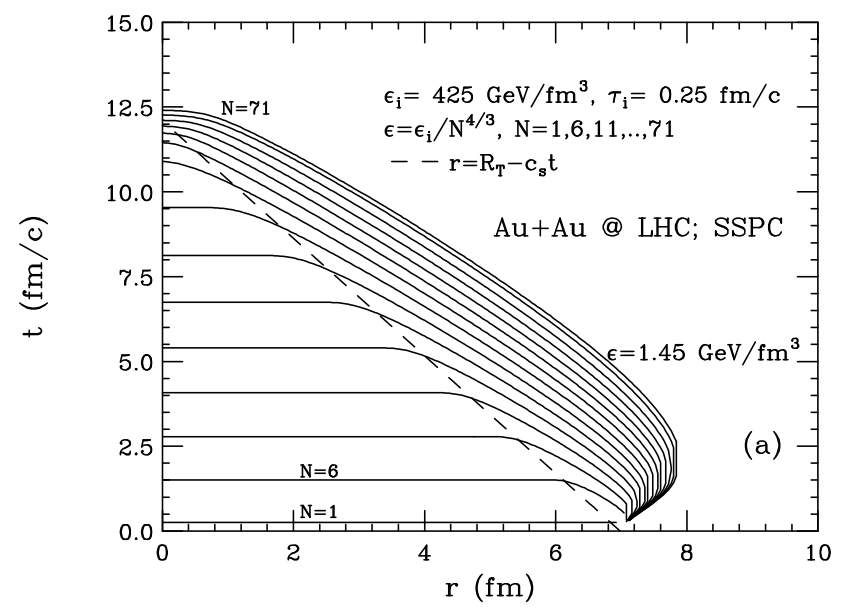



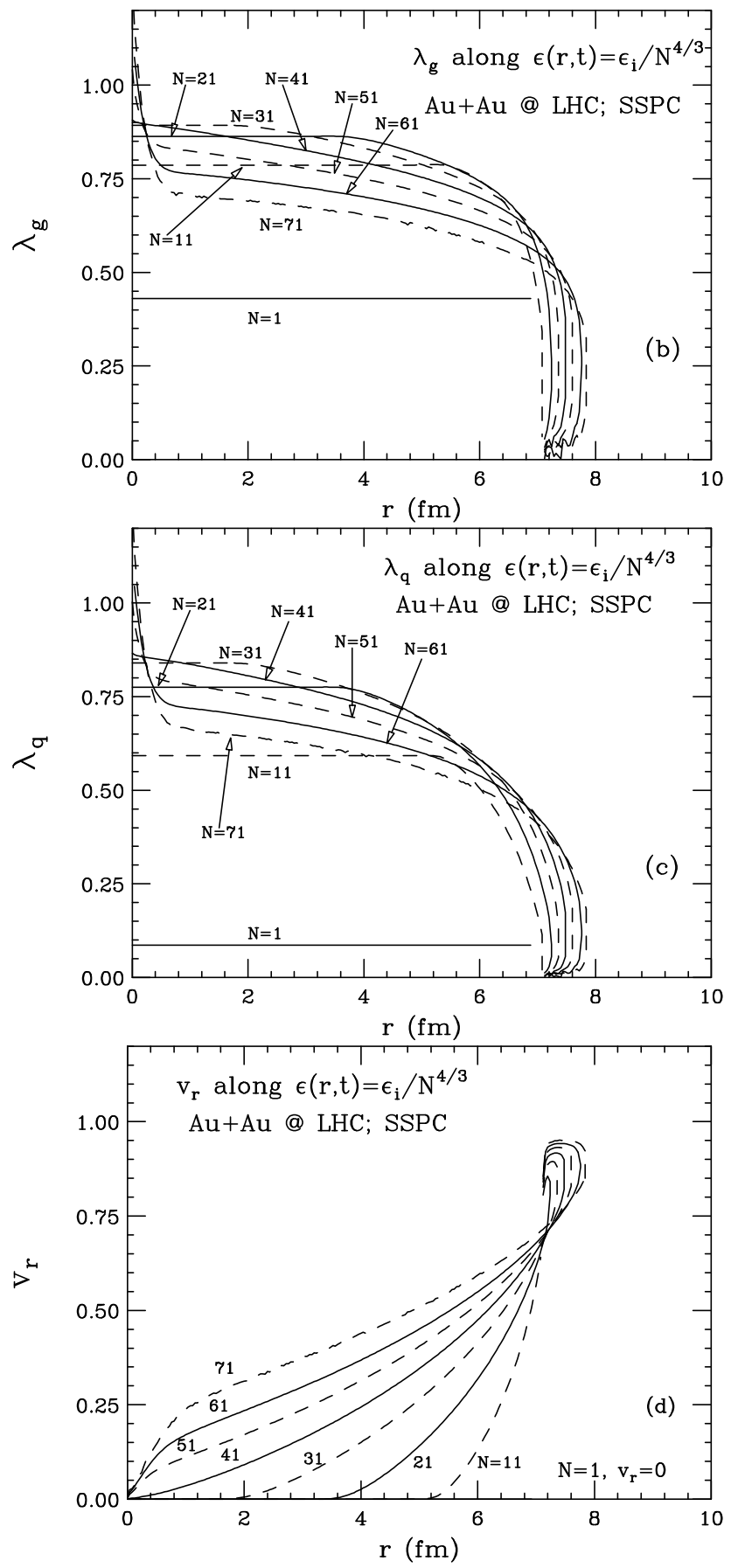

FIG. 3. (a)-(d): Same as Fig. 1, at LHC.

The same effects pertaining to the collective transverse flow are observed as at the lower RHIC energy, but they are more pronounced. Due to the longer lifetime of the plasma phase at LHC energies, the effects of the transverse expansion are generally more dramatic. The rarefaction wave reaches the center after about $12 \mathrm{fm} / c$, and all of the matter participates in the transverse flow at the moment of hadronization. The more rapid cooling of the system is also indicated by the decreasing separation of the contours at later times. This affects the chemical equilibration process in several competing ways.
We note that, as before, the quark fugacities lag behind the gluon fugacities at all times and all radial distances. This is not surprising, considering the lower starting value for $\lambda_{q}$. We also see again that the fugacities in the interior, at first, increase with passage of time. However, beyond $N=31$, corresponding to about $8 \mathrm{fm} / c$ near the center, when the transverse flow has been substantially developed, the fugacities begin decreasing again, except at $r=0$, where they continue to increase, as all the radial derivatives in (11) are small there.

Let us pause to understand this unexpected feature. According to (6), the evolution of the parton densities is governed by $\partial_{\mu}\left(n u^{\mu}\right)$ which consists of two terms: $u^{\mu} \partial_{\mu} n$ gives the rate of change of the density in the comoving frame, and $n \partial_{\mu} u^{\mu}$ describes the rate of change of the density due to the expansion of the fluid element in the comoving frame [19]. Once the transverse expansion of the fluid starts developing, the second term and also the radial derivatives in (11) grow very rapidly and drive the system away from chemical equilibrium.

We thus see that two mechanisms potentially contribute to the depletion of the parton densities: expansion and flow. Let us concentrate on the consequences of the latter, as the consequences of the expansion alone are easy to visualize. Figure 4 depicts a fluid element at a distance $r$ from the axis at some instant of time, in a central collision for the conditions of: (a) no flow, (b) uniform transverse flow, and (c) a differential transverse flow with the velocity field $v_{r}(r, t)$ as obtained in the present work. When there is no flow, the thermal motion of the partons brings the same number of partons into the volume element as are leaving it on all sides. Thus any production of new partons due to the chemical reactions in the volume element will increase the fugacities, which will hence rise monotonically since the right hand sides of the master equations $(9,11)$ remain positive [4, 16].

(a)

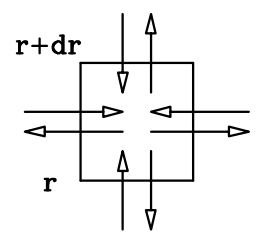

No Flow

$r$

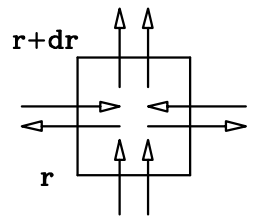

$\mathrm{v}_{\mathrm{r}}=$ Cons

(c)

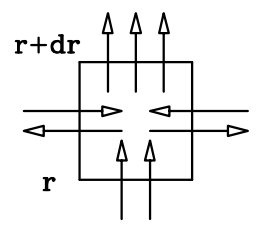


FIG. 4. Depletion of partons for, (a) no flow, (b) uniform flow, and (c) flow with velocity gradient in a fluid element at $r$.

Next consider the condition of a uniform transverse flow, which is sometimes used as a simplifying assumption 20]. This scenario still does not produce a net outflow of partons from the volume element, because the loss of partons on one side is exactly balanced by the gain of partons flowing in from the opposite side. Thus, if there is a creation of new partons due to chemical reactions then, as before, the fugacities will increase with time.

However, when the transverse velocity is driven by the pressure gradient as in our calculation, $v_{r}(r+d r)>v_{r}(r)$. Then the velocity gradient will ensure that more partons are leaving the fluid element at $r+d r$ than are entering at $r$. This leads to an additional depletion of the parton density, beyond the dilution caused by the overall expansion. If the velocity gradient is sufficiently large, the production of new partons by the chemical reactions cannot cope with the depletion and the fugacities will start to fall. The richness of this scenario is completely absent when the simplifying assumptions of no-flow or of a uniform transverse flow are made.

Let us return to the discussion of the consequences of the transverse flow on the momentum distribution of the partons at LHC energies. In Figs. 5a-b, we show the evolution of the gluon and quark momentum distributions over the course of the expansion. We see that the distribution at later times is strongly affected by the flow. It is again apparent that there is an approximate balance between the decreasing temperature and the increasing transverse velocity which keeps the parton distributions at high $p_{T}$ largely independent of $N$. This is reminiscent of a similar behavior observed in pion spectra when transverse flow is introduced [21].

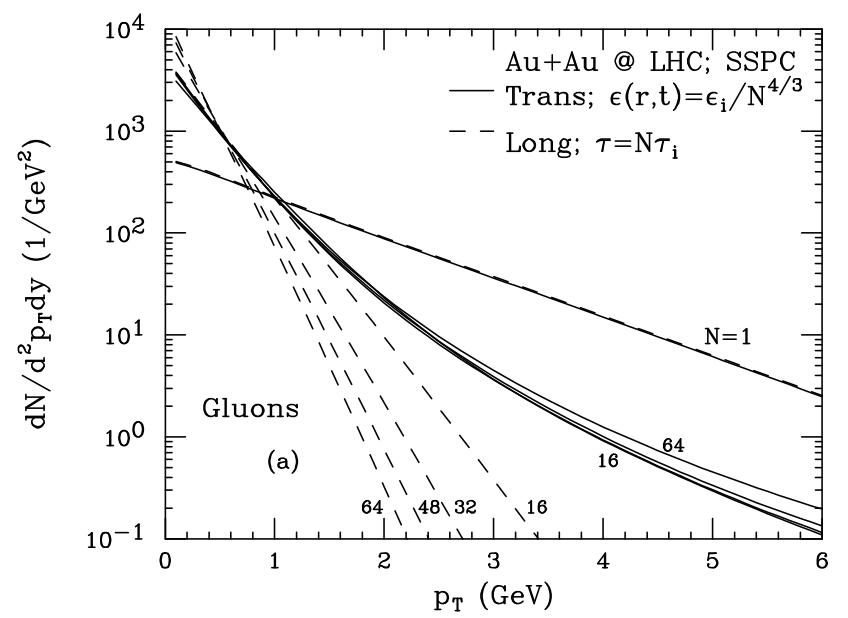

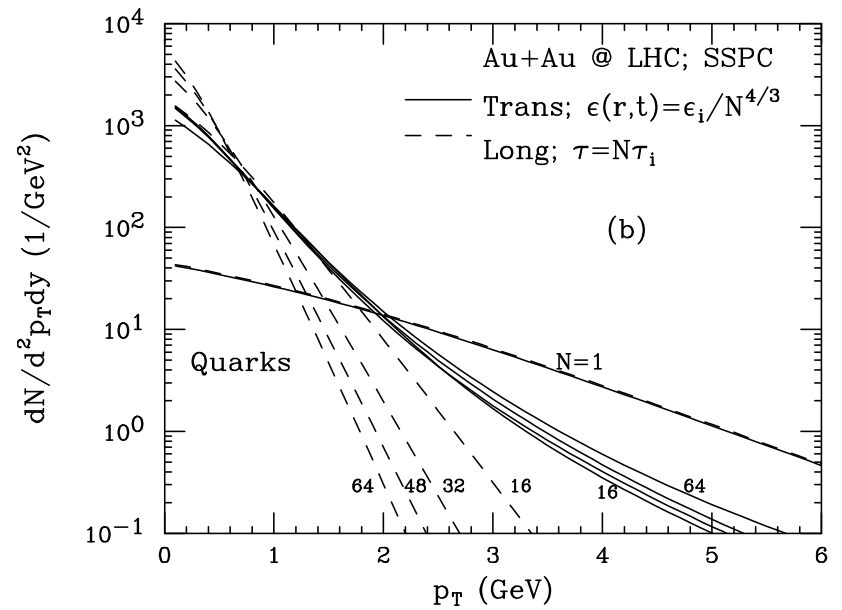

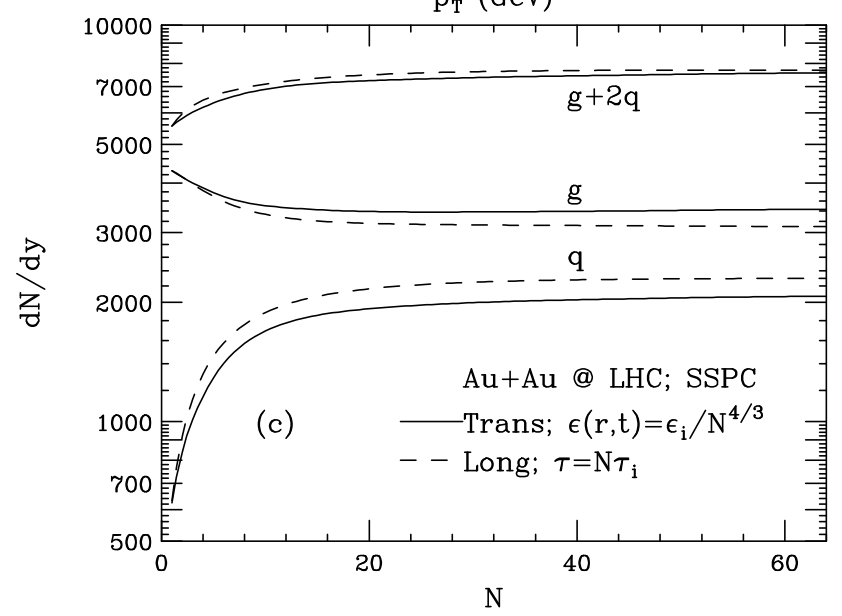

FIG. 5. (a)-(c) Same as Fig. 2, for LHC.

Figure $5 \mathrm{c}$ shows our results for the evolution of the parton multiplicity. We see a significant production of quarks and antiquarks, in fact an increase by a factor of about 2.5, and a decrease in the number of gluons by about $25 \%$, as a result of the chemical reactions in the plasma. This results in a net increase of the parton multiplicity by approximately $40 \%$. As before at RHIC energies, we find that the transverse flow does not strongly affect the net production of partons, although it influences the individual abundances of gluons and quarks.

It is probably difficult to probe the off-chemical equilibrium nature of the light quark and gluon fractions in the plasma. However, we can think of a practical way of testing the chemical equilibrium in the cases of heavier quark flavors, viz., strange, charm, and even bottom quarks, by inferring this information from the ratios of the (valence) quark content of the emitted hadrons 22, 23]. We can analogously define the ratio of heavier quarks to light quarks as

$$
\mathcal{R}=\frac{\langle Q+\bar{Q}\rangle}{0.5[\langle u+\bar{u}\rangle+\langle d+\bar{d}\rangle]}
$$

where $Q$ stands for one of the heavy quarks. It is not unlikely that this ratio can be largely determined from the final production of strange, charm, or bottom mesons 
and pions [5].

Simulations of the pure SU(3) lattice gauge theory have shown that the speed of sound drops significantly as the temperature approaches the phase transition temperature from above [24]. If this observation carries over to full QCD, our results could be modified. A lower speed of sound generally leads to a reduced production of transverse flow and, hence, to an increased life-time of the QGP. This effect should be especially pronounced at RHIC energies 25].

Can we devise means for identifying the large transverse flow velocities predicted here? As mentioned earlier, the life-time of the mixed phase, which follows the QGP phase, gets shorter if the transverse velocity increases. Because the transverse flow will remain frozen in the mixed phase, the final hadronic flow pattern should be a rather reliable measure of the transverse velocity established during the QGP phase. In a forthcoming publication, we shall report on the effect of the transverse flow on the $p_{T}$-spectra of charmed hadrons which are considered to be good probes of flow due to their large mass. In the next section we look at the spectra of single photons and lepton pairs and see how far could they be affected by these consideration.

\section{Other Initial Conditions}

Let us now briefly examine the consequences of using different initial thermalization conditions. We only present the results for the hadronization contour, $\epsilon(r, t)=1.45 \mathrm{GeV} / \mathrm{fm}^{3}$, and for the gluon and quark fugacities attained along this contour for RHIC (Fig. 6a-b) and LHC (Fig. 7a-b) energies. We select several initial conditions obtained by modifying predictions of the HIJING model, as listed in Table I. The original prediction of the HIJING model [11] is denoted as I. The second set (II) of initial conditions is obtained by increasing the initial fugacities, and hence the energy density, by a factor four. The third set (III) is obtained by retaining the fugacities of the second set, but decreasing the temperature to account for the production of soft partons from the color field, by some arbitrary amount. It is of interest to recall that the Parton Cascade Model of Geiger [6] predicts an energy density of about $120 \mathrm{GeV} / \mathrm{fm}^{3}$, and a temperature of about $590 \mathrm{MeV}$, at $\tau=0.25 \mathrm{fm} / c$ in central collisions of two gold nuclei at RHIC energies.
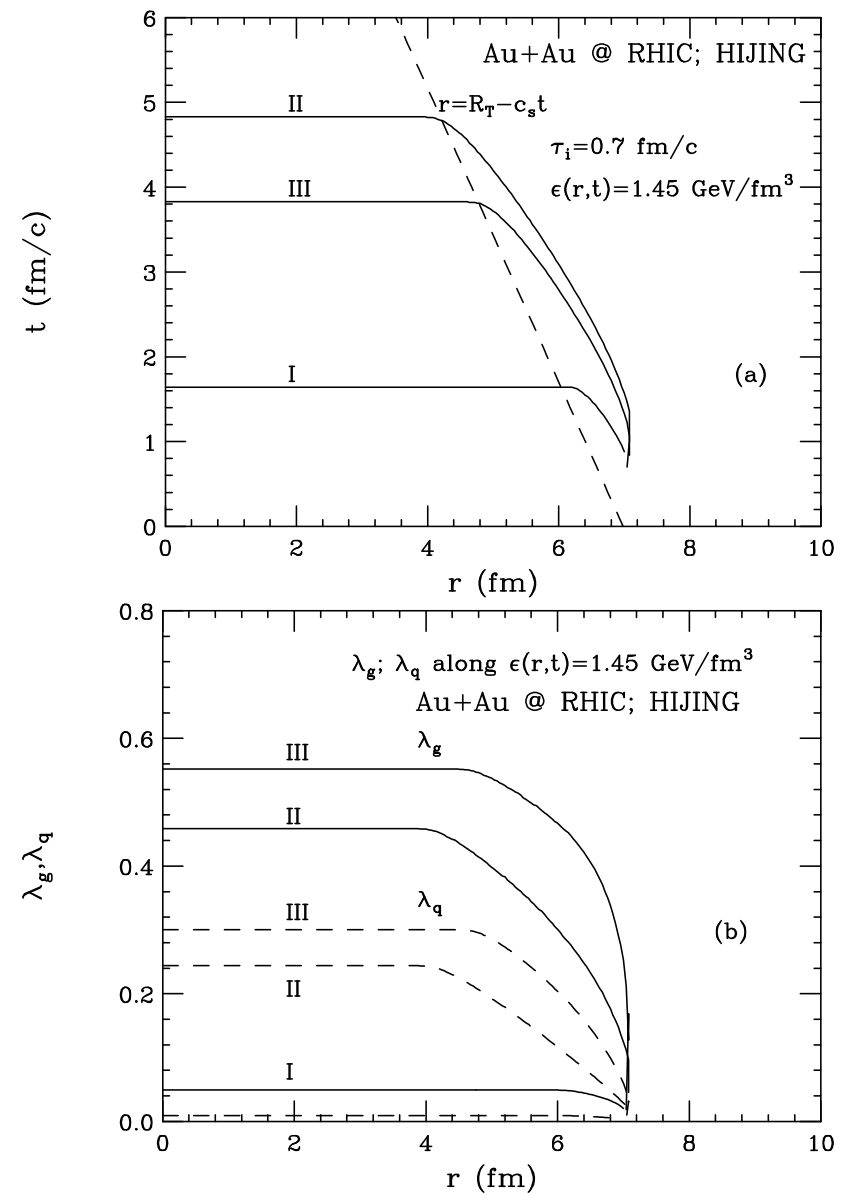

FIG. 6. (a) Constant energy density contours for $\epsilon=1.45$ $\mathrm{GeV} / \mathrm{fm}^{3}$ for the initial conditions obtained from HIJING, given in Table I. (b) Gluon and quark fugacities along these contours.

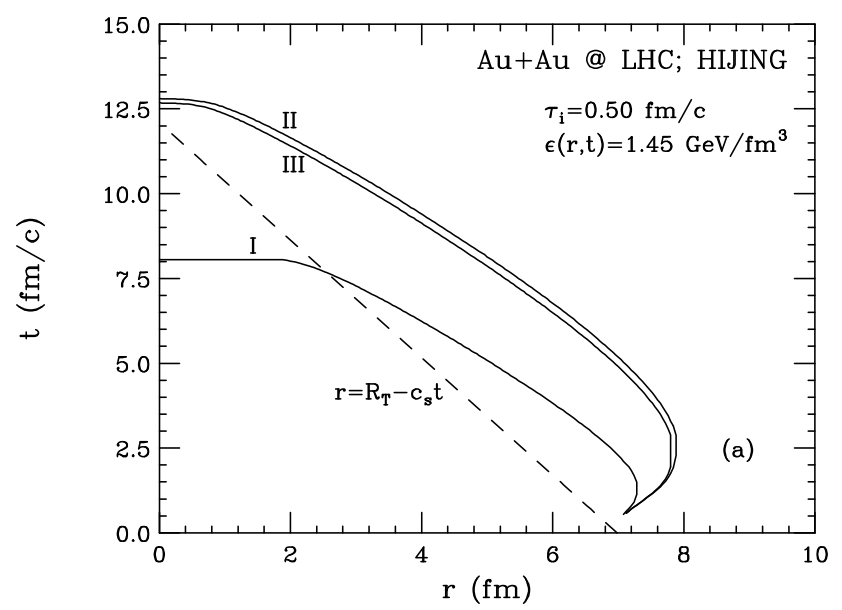




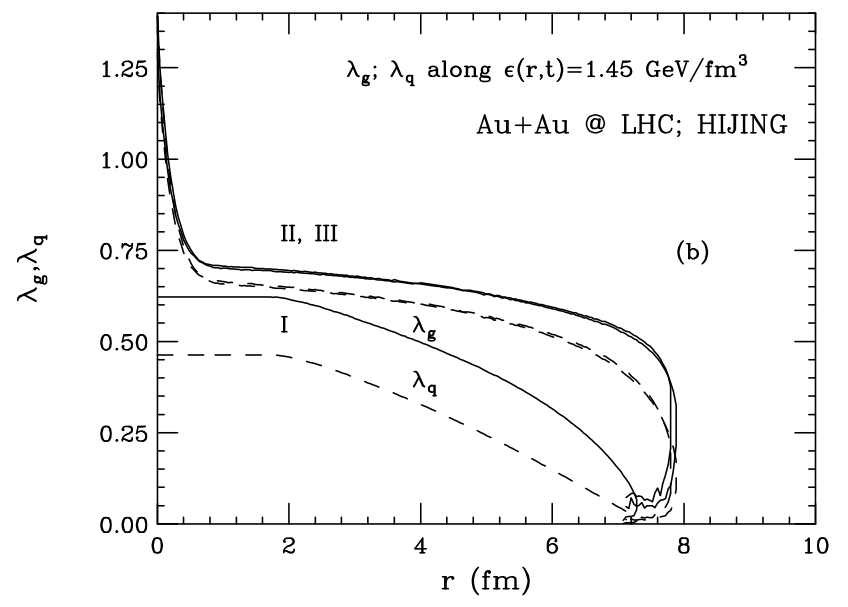

FIG. 7. Same as Fig. 6, for LHC.

If we use the original predictions (I) of the HIJING model, then the life-time of the QGP phase is very small (less than $2 \mathrm{fm} / c$ ), and the matter at the moment of hadronization is extremely dilute compared with chemically equilibrated matter. Changing the initial fugacities by a factor of 4 increases the life-time of the QGP phase considerably and also brings the system close to chemical equilibrium. Note that the initial fugacities differ by one order of magnitude between the conditions I and III at RHIC energies. Note also that the final fugacities at large $r$ are quite different, even though the energy densities are identical, signaling very different temperatures at the end of the QGP phase.

Results for LHC energies indicate the effect of transverse expansion for $r \geq 4 \mathrm{fm}$, for the original predictions of HIJING, marked as I, and the entire fluid is affected by the flow for the other initial conditions, which assume much larger initial energy densities and fugacities. The final fugacities for the conditions II and III are essentially identical and show the effect of evolution away from chemical equilibrium, as the flow velocity gets large. The transverse dimension of the system grows by almost $1 \mathrm{fm}$ during the QGP phase itself for the initial conditions II and III. Since the fugacities shown in these two figures are for the same energy density $\epsilon_{f}$, we note that the final temperature for the standard case (I) will be much larger than in the scenarios II and III.

\section{THERMAL PHOTONS AND LEPTON PAIRS}

\section{A. Photon Spectra}

Thermal photons and lepton pairs are primary probes of all the stages of the nuclear reaction. They are also expected to carry valuable imprints of the transverse expansion of the system. Thermal photons from the quarkgluon plasma have their origin in the Compton $(q g \rightarrow q \gamma)$ and annihilation processes $(q \bar{q} \rightarrow g \gamma)$. It is useful to recall the expressions for the rates for these contributions for a non-equilibrated quark-gluon plasma [26]. For the approximations of the fugacities that we have used, the Compton process contributes with the rate

$$
\begin{aligned}
E \frac{d N_{\gamma}}{d^{3} p d^{4} x}=\frac{2 \alpha \alpha_{s}}{\pi^{4}} \lambda_{q} \lambda_{g} T^{2} e_{q}^{2} \exp (-E / T) & \\
& {\left[\ln \left(\frac{4 E T}{k_{c}^{2}}\right)+\frac{1}{2}-C\right], }
\end{aligned}
$$

and the rate of the radiative annihilation process is,

$$
\begin{aligned}
E \frac{d N_{\gamma}}{d^{3} p d^{4} x}=\frac{2 \alpha \alpha_{s}}{\pi^{4}} \lambda_{q} \lambda_{\bar{q}} T^{2} e_{q}^{2} \exp (-E / T) & \\
& {\left[\ln \left(\frac{4 E T}{k_{c}^{2}}\right)-1-C\right] . }
\end{aligned}
$$

Here $C=0.577721 \ldots, e_{q}$ is the electric charge of the quark and the parameter $k_{c}$ is related to the thermal mass of the quarks in the medium.

Recalling that our plasma is gluon-rich and quarkpoor, we anticipate that the yield of the thermal photons would be dominated by the Compton contribution. In Figs. 8 and 9, we give our results for RHIC and LHC energies, respectively, for the initial conditions obtained from the SSPC [10]. We show the Compton and annihilation contributions separately for the scenario involving transverse expansion. The results for longitudinal expansion are given for comparison.

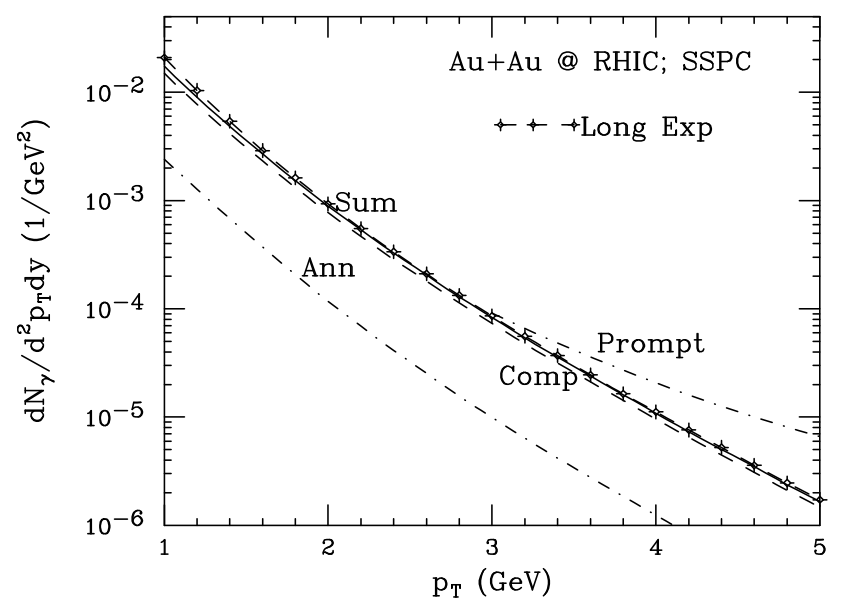

FIG. 8. Distribution of thermal photons from the QGP phase at RHIC. The Compton and annihilation yields, and their sum are shown for the case with transverse expansion. Results are also given for a purely longitudinal flow. Prompt photons, whose production is governed by structure functions, are seen to dominate the yield for $p_{T}>3-4 \mathrm{GeV}$. 


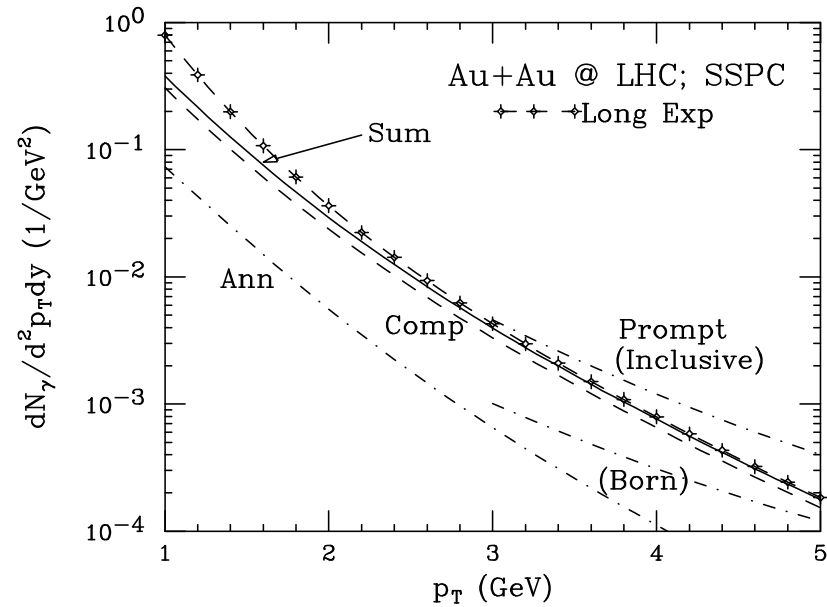

FIG. 9. Same as Fig. 8 at LHC. The prompt photons from fragmentation of quark jets are also shown.

We see that the transverse expansion of the fluid leaves the thermal photon production from the QGP at RHIC energies essentially unchanged for $p_{T}>1 \mathrm{GeV}$. The reduction of the thermal photon production at lower $p_{T}$ will be difficult to identify, as it will be overshadowed by photons from hadronic reactions and hadronic decays. We also see that prompt photons [27] will dominate the yield beyond $p_{T} \geq 4 \mathrm{GeV}$. At first this result looks surprising, in view of the large modifications of the final parton spectra seen earlier (cf. Figs. 2 and 5). However, note that the rates for photon emission (16,17) carry an additional weight factor of $T^{2}$, which suppresses the contributions from later times. Note also that the time available for radiation at a given temperature is significantly reduced by the transverse expansion. Overall, we conclude that the thermal photon production from the plasma phase is remarkably insensitive to the transverse expansion at RHIC energies.

The production of thermal photons with large $p_{T}$ remains essentially unchanged at LHC, as well, as they have origin in the early hot stages, when the flow effects are still small. On the other hand, the production of low $p_{T}$ photons decreases considerably, due to reduction in the space-time volume occupied by colder matter in the presence of transverse expansion. While the prompt photon production [27] may remain lower than the thermal photon yield up to $p_{T}=5 \mathrm{GeV}$, the background contribution of photons fragmented off high- $p_{T}$ quark jets is large.

\section{B. Lepton Pair Spectra}

The mass distribution of lepton pairs obtained from quark annihilation are given in Figs. 10 and 11, for RHIC and LHC energies, respectively, without and with transverse expansion. The Drell-Yan contribution [28] is also shown. We again see a negligible effect of the flow on the mass distribution of lepton pairs at RHIC energies, but a factor of 2-3 reduction is seen in the yield at LHC energies at invariant masses of $1-2 \mathrm{GeV}$, due to the reduced life-time of the plasma. We also note that the Drell-Yan contribution dominates the yield beyond $M \geq 4 \mathrm{GeV}$ at LHC, whereas this zone shifts to much smaller $M$ at RHIC. This is primarily due to very low quark densities of the plasma.

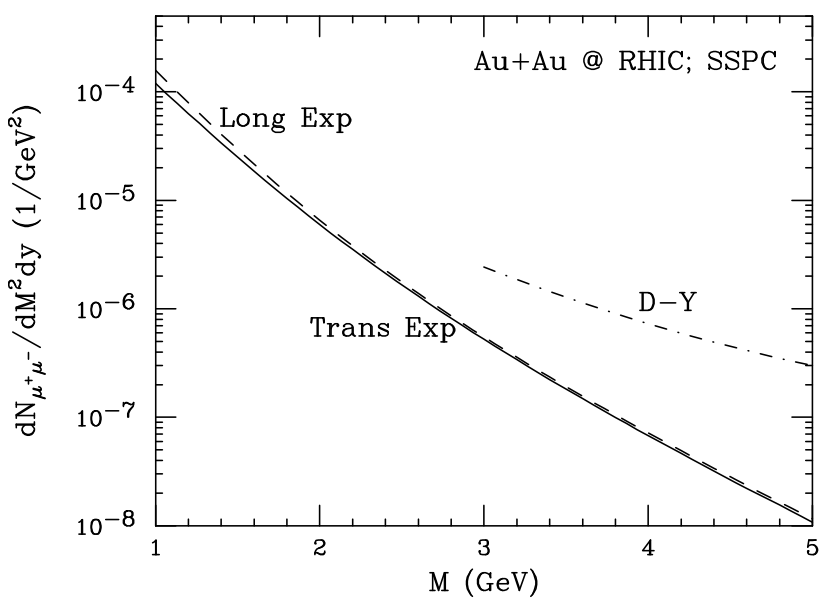

FIG. 10. Mass distribution of thermal lepton pairs from the QGP phase at RHIC with (solid curves) and without (dashed curves) transverse flow. The Drell- Yan contribution is also given.

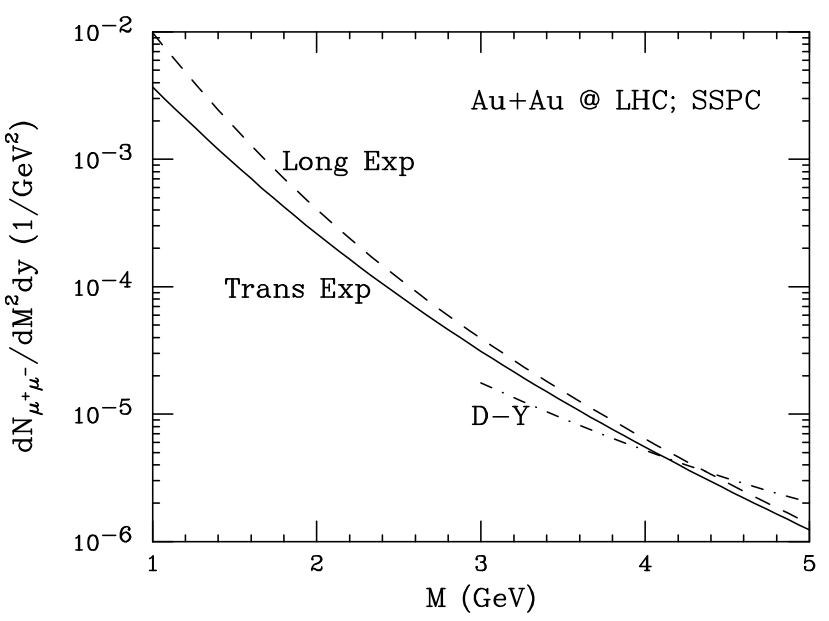

FIG. 11. Same as Fig. 10 for LHC.

These small deviations in the production of thermal photon and lepton pairs due to the transverse expansion are rather astounding. We have already noted that this has its origin in the strong temperature dependence of the production rates, combined with the accelerated cooling of a transversely expanding plasma. This raises the question whether it is at all possible to identify the presence of transverse flow from the lepton pair spectra. Recall that the transverse mass distribution of the lepton pairs from the QGP in presence of a flow will be given by, 


$$
\begin{aligned}
\frac{d N_{\mu^{+} \mu^{-}}}{d M^{2} d^{2} M_{T} d y}=\frac{\alpha^{2}}{2 \pi^{3}} \lambda_{q} \lambda_{\bar{q}} e_{q}^{2} \int \tau d \tau r d r \\
I_{0}\left(\frac{\gamma v_{r} p_{T}}{T}\right) K_{0}\left(\frac{\gamma M_{T}}{T}\right) .
\end{aligned}
$$

In the absence of a flow, this reduces to

$$
\frac{d N \mu^{+} \mu^{-}}{d M^{2} d^{2} M_{T} d y}=\frac{\alpha^{2}}{4 \pi^{3}} \lambda_{q} \lambda_{\bar{q}} e_{q}^{2} R_{T}^{2} \int \tau d \tau K_{0}\left(\frac{M_{T}}{T}\right),
$$

which scales with $M_{T}$ in a characteristic manner which is known as $M_{T^{-}}$scaling [29, 30]. This scaling will be violated in the presence of a transverse flow [30].

In Figs. 12 and 13 we present our predictions for the ratios of the transverse mass distributions for $M=1$ $\mathrm{GeV}$ to that for $M=2,3$, and $4 \mathrm{GeV}$ at RHIC and LHC energies, respectively. As indicated earlier these ratios are identically one for a purely longitudinal expansion. The deviations predicted here are not large, but we hope that they will be observable in the high statistics data that will be forthcoming from RHIC and LHC. Additional information about the parton fugacities is contained in the absolute yields of the photon and lepton pair spectra, because the thermal photon yield is dominated by the Compton processes $\left(\propto \lambda_{g} \lambda_{q}\right)$, whereas the lepton pair yield is mainly due to quark-antiquark annihilation $\left(\propto \lambda_{q}^{2}\right)$.

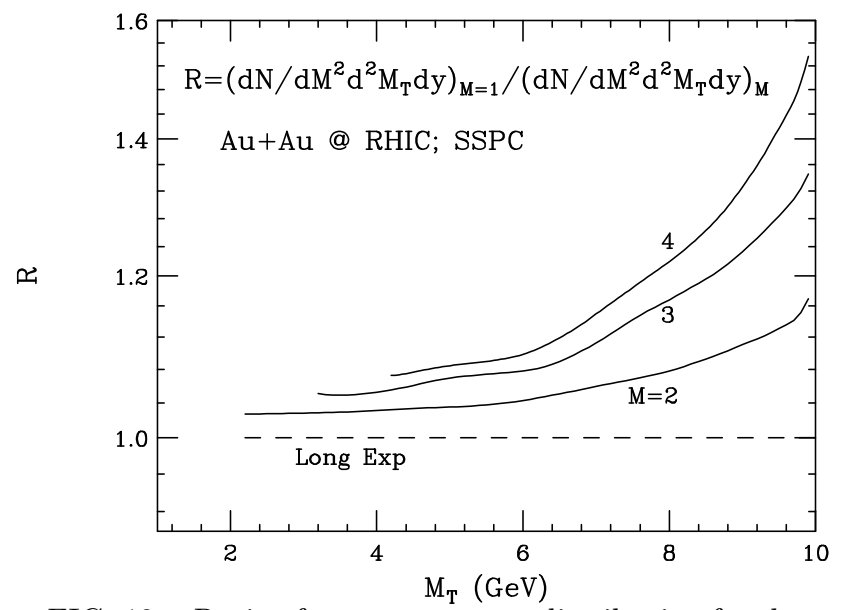

FIG. 12. Ratio of transverse mass distribution for thermal lepton pairs for $M=1 \mathrm{GeV}$ to that for a given $M$. In absence of flow, it is identically unity.

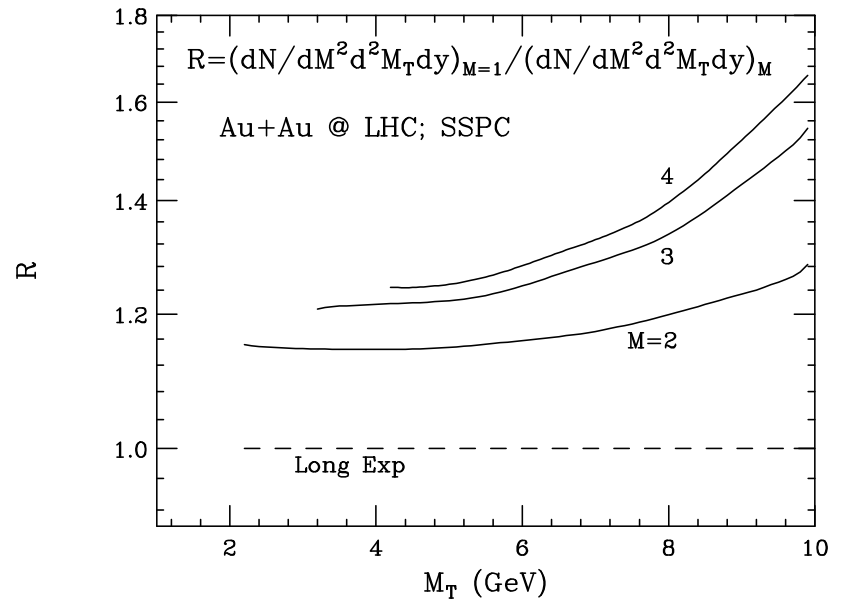

FIG. 13. Same as Fig. 12 at LHC.

\section{SUMMARY}

We have studied the evolution of an equilibrating and transversely expanding quark-gluon plasma, with initial conditions that have been obtained either from the self-screened parton cascade model or from the HIJING model. We have compared the results from a boost invariant longitudinal, as well as from a transverse expansion of the plasma. The velocity gradients generated due to flow were found to affect the individual concentrations of the quarks, antiquarks, and gluons, without affecting the total final entropy.

Large transverse velocities are developing by the end of the QGP phase, especially at LHC energies. The flow is also seen to drive the system away from chemical equilibrium. As the process of chemical equilibration accelerates cooling, and as the rates of emission of photons and lepton pairs depend strongly on the temperature, the final spectra are only slightly affected by them. This has its origin in the fact that photons and lepton pairs originate from the early hot stage when the flow is still small. However, the transverse flow is seen to introduce a slight violation of the so-called $M_{T}$ scaling of the lepton pair spectra. Likely consequences on the post-QGP epoch could be a copious production of low $p_{T}$ pions from the fragmentation of residual gluons. We also note that the temperature of the matter at the end of the so-called QGP phase, may be higher at the surface than in the center of the reaction volume.

\section{ACKNOWLEDGMENTS}

We acknowledge useful comments from Pradip Kumar Roy, Sourav Sarkar, and Bikash Sinha. This work was supported in part by a grant from the U.S. Department of Energy (DE-FG02-96ER40945). 
[1] J.W. Harris and B. Müller, Annu. Rev. Nucl. Part. Science 46, 71 (1996).

[2] K. Geiger and B. Müller, Nucl. Phys. B369, 600 (1992); K. Geiger, Phys. Rep. 258, 376 (1995).

[3] K.J. Eskola and X.N. Wang, Phys. Rev. D49, 1284 (1994).

[4] T. S. Biro, E. van Doorn, B. Müller, M. H. Thoma, and X. N. Wang, Phys. Rev. C 48, 1275 (1993).

[5] K. Geiger and J. I. Kapusta, Phys. Rev. D 47, 4905 (1993).

[6] K. Geiger, Phys. Rev. D 48, 4129 (1993).

[7] J. Alam, S. Raha, and B. Sinha, Phys. Rev. Lett. 73, 1895 (1994).

[8] X. M. H. Wong, Nucl. Phys. A 607, 442 (1996).

[9] D. K. Srivastava, M. G. Mustafa, and B. Müller, hepph/9608424 $)$, submitted to Phys. Rev. Lett.

[10] K. J. Eskola, B. Müller, and X. N. Wang, Phys. Lett. B 374, 20 (1996).

[11] X.-N. Wang and M. Gyulassy, Phys. Rev. D 44, 3501 (1991); Comput. Phys. Commun. 83, 307 (1994).

[12] X.-M. Xu, D. Kharzeev, H. Satz, and X.-N. Wang, Phys. Rev. C 53, 3051 (1996).

[13] $\epsilon_{f}=1.46 \mathrm{GeV} / \mathrm{fm}^{3}$ corresponds to the energy density for a fully equilibrated QGP at $T \approx 160 \mathrm{MeV}$.

[14] H. von Gersdorff, L. McLerran, M. Kataja, and P. V. Ruuskanen, Phys. Rev. D 34, 794 (1986).

[15] J. D. Bjorken, Phys. Rev. D 27, 140 (1983).

[16] K. J. Eskola, B. Müller, and X.-N. Wang, DUKE-TH-96120.

[17] F. Cooper and G. Frye, Phys. Rev. D 10, 186 (1974).

[18] P. V. Ruuskanen, Acta Phys. Pol. B 18, 551 (1986).

[19] K. Kajantie, M. Kataja, and P. V. Ruuskanen, Phys. Lett. B 179, 153 (1986).

[20] B. Kämpfer and O. P. Pavlenko, Z. Phys. C 62, 491 (1994); Phys. Rev. C 49, 2716 (1994).

[21] J. Cleymans, K. Redlich, and D. K. Srivastava, GSIPreprint-96-42, UCT-TP 231/96, Phys. Rec. C (in press).

[22] J. Cleymans and H. Satz, Z. Phys. C 57, 135 (1993).

[23] P. Braun-Munzinger, J. Stachel, J. P. Wessels, and N Xu, Phys. Lett. B 344, 43 (1995).

[24] G. Boyd, J. Engels, F. Karsch, E. Laermann, C. Legeland, M. Lütgemeier, B. Petersson, Nucl. Phys. B 469, 419 (1996).

[25] D.H. Rischke and M. Gyulassy, Nucl. Phys. A 597, 701 (1995) and Columbia University preprint nuclth/9606039).

[26] M. T. Strickland, Phys. Lett. B 331, 245 (1994).

[27] J. Cleymans, E. Quack, K. Redlich, and D. K. Srivastava, Int. J. Mod. Phys. A 10, 2941 (1995).

[28] S. Gavin, R. Kaufmann, S. Gupta, P. V. Ruuskanen, D. K. Srivastava, and R. L. Thews, Int. J. Mod. Phys. A 10, 2961 (1995).

[29] M. Asakawa, C. M. Ko, and P. Levai, Phys. Rev. Lett. 70, 398 (1993).

[30] D. K. Srivastava, J. Pan, V. Emel'yanov, and C. Gale, Phys. Lett. B 329, 157 (1994).
TABLE I. Initial conditions for the hydrodynamical expansion phase in central collision of two gold nuclei at BNL RHIC and CERN LHC energies from SSPC and HIJING models.

\begin{tabular}{l|c|c|c|c|c|}
\hline \hline Energy & $\tau_{i}$ & $T_{i}$ & $\lambda_{g}^{(i)}$ & $\lambda_{q}^{(i)}$ & $\epsilon_{i}$ \\
& $(\mathrm{fm} / \mathrm{c})$ & $(\mathrm{GeV})$ & - & - & $\left(\mathrm{GeV} / \mathrm{fm}^{3}\right)$ \\
\hline SSPC & & & & & \\
RHIC & 0.25 & 0.668 & 0.34 & 0.064 & 61.4 \\
LHC & 0.25 & 1.02 & 0.43 & 0.082 & 425 \\
HIJING & & & & & \\
RHIC, I & 0.7 & 0.55 & 0.05 & 0.008 & 4.0 \\
RHIC, II & 0.7 & 0.55 & 0.20 & 0.032 & 15.8 \\
RHIC, III & 0.7 & 0.40 & 0.53 & 0.083 & 11.7 \\
LHC, I & 0.5 & 0.82 & 0.124 & 0.02 & 48.6 \\
LHC, II & 0.5 & 0.82 & 0.496 & 0.08 & 194 \\
LHC, III & 0.5 & 0.72 & 0.761 & 0.118 & 176 \\
\hline \hline
\end{tabular}

